\title{
Liouville Theorems for Fuchsian-Type Operators on the Heisenberg Group
}

\author{
M. R. Lancia and M. V. Marchi
}

\begin{abstract}
We prove some Liouville theorems for a degenerate elliptic operator whose principal part is given in divergence form with respect to the Heisenberg vector fields and lower terms satisfy Fuchsian-type conditions with respect to the intrinsic norm.

Keywords: Asymptotic behaviour, positive solutions, degenerate elliptic operators, Fuchsiantype operators, Heisenberg operators
\end{abstract}

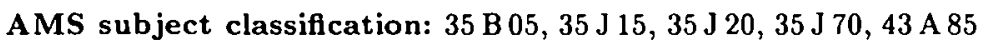

\section{Introduction}

In this paper we study the asymptotic behavior of the local solutions of the equation

$$
L u=0
$$

where the operator $L$ is given by

$$
L u=-\sum_{i, j=1}^{2 n} X_{j}^{*}\left(a_{i j}(x) X_{i} u+d_{j}(x) u\right)+\sum_{i=1}^{2 n} b_{i}(x) X_{i} u+c(x) u
$$

and $X_{j}$ are the Heisenberg vector fields in $\mathbb{R}^{2 n+1}, X_{j}^{*}$ is the $L^{2}$-adjoint of $X_{j}$. The operator $L$ is assumed to be uniformly subelliptic and weakly Fuchsian with respect to the intrinsic dilations. More precisely we will assume the following:

(A) $a_{i j}$ are measurable functions on $\mathbb{R}^{2 n+1}$, and there exist positive constants $\mu \leq M$ such that

$$
\mu|\xi|^{2} \leq \sum_{i, j=1}^{2 n} a_{i j}(x) \xi_{i} \xi_{j} \leq M|\xi|^{2}
$$

for all $x \in \mathbb{R}^{2 n+1}$ and $\xi \in \mathbb{R}^{2 n}$.

M. R. Lancia: Università di Roma "La Sapienza", Dipartimento di Metodi e Modelli Matematici-per le Scienze Applicate, Via A. Scarpa 10, 00161 Roma, Italia.

M. V. Marchi: Università di Roma "La Sapienza", Dipartimento di Matematica, Piazzale A. Moro 2, 00185 Roma, Italia 
(B) There exist $q>2 n+2, \Lambda>0,0<a<1<b$ and a sequence $R_{k} \nearrow+\infty$ such that, for every $k \geq 1$,

$$
\rho b_{i} \in L^{q}\left(A_{k}\right), \quad \rho d_{i} \in L^{q}\left(A_{k}\right) \quad(1 \leq i \leq n), \quad \rho^{2} c \in L^{\frac{q}{2}}\left(A_{k}\right)
$$

and

$$
\left\|\rho b_{i}\right\|_{L^{\natural}\left(A_{k}\right)} \leq \Lambda\left|A_{k}\right|^{\frac{1}{q}}, \quad\left\|\rho d_{i}\right\|_{L^{q}\left(A_{k}\right)} \leq \Lambda\left|A_{k}\right|^{\frac{1}{q}} \quad(1 \leq i \leq 2 n)
$$

where $\rho$ is the norm defined in (2.3), intrinsically associated with the vector fields $X_{j}$, $A_{k}$ are the open annuli:

$$
A_{k}=\left\{x \in \mathbb{R}^{2 n+1}: a R_{k}<\rho(x)<b R_{k}\right\}
$$

and $\left|A_{k}\right|$ denotes the Lebesgue measure of $A_{k}$.

We will also assume that $L$ satisfies the Maximum Principle (see Section 3).

The topics include the following results.

Proposition 4.4. For every two positive local solutions $u$ and $v$ of $L$ in $\mathbb{R}^{2 n+1} \backslash B_{R}$, there exists

$$
\lim _{\rho(x) \rightarrow+\infty} \frac{u(x)}{v(x)} .
$$

where $B_{R}$ denotes the intrinsic ball defined in (2.8).

Theorem 4.5 (Liouville Theorem). There exists a unique, up to a constant, positive local solution of the equation (1.1) in $\mathbb{R}^{2 n+1}$.

When $L$ is a Fuchsian operator (see condition (4.11)), we can prove also the following statement.

Theorem 4.7 (Liouville Property). Every bounded local solution of the equation (1.1) in $\mathbb{R}^{2 n+1}$. is of constant sign.

Our method to study the asymptotic behavior of the solutions is to show, as in [9, $10,14,15]$, that the positive solutions satisfy a uniform Harnack inequality. The local Harnack inequality for the operator defined in (1.2) has been proved by M. Biroli and U. Mosco in [3], under the assumptions $a_{i}$, symmetric and $b_{i} \equiv d_{i} \equiv c \equiv 0$, in the more general context of the Dirichlet forms. In [13] there is given a local Harnack inequality for the operator (1.2), where $X_{j}$ are Hörmander vector fields.

In order to have a uniform Harnack inequality we need some control on the growth of the lower terms (see, for example, $[1,5,14,15])$. In the classical case this control is given by Fuchsian assumptions, related to the homogeneity of the differential operators $\frac{\partial}{\partial x_{i}}$ with respect to the usual dilations. In our case the assumptions on the growth of the lower terms reflect the non-isotropic character of the fields $X_{j}$. The Liouville theorems for the Heisenberg Laplacian has been proved by B. Gaveau [8]. For the nonlinear case see also [2] and [7]. 
The plan of the paper is the following. In Section 2 we recall the main properties of the Heisenberg group and of the associated Sobolev spaces. In Section 3 we recall some results about the operator $L$ in bounded domains, we discuss the Maximum Principle and the existence and uniqueness for the Dirichlet problem, and we prove an existence theorem for the equation (1.1) in $\mathbb{R}^{2 n+1}$. In Section 4 , by assuming $L$ to be a weakly Fuchsian operator, we prove the existence of the limit (1.6) and Liouville's theorems.

Finally we recall that all the previous results still hold for the operator

$$
\widetilde{L} u=L u+g(x) X_{0} u
$$

where $L$, defined in (1.2), is assumed to satisfy assumptions (A) and (B) and, in addition, the following one:

(C) $X_{0}$ is a bounded smooth vector field in $\mathbb{R}^{2 n+2}$, i.e.

$$
X_{0}=\sum_{i=1}^{2 n} \sigma_{i} X_{i}+\sigma_{2 n+1} T \quad\left(\sigma_{i} \in C^{\infty}\left(\mathbb{R}^{2 n+1}\right), i=1, \ldots, 2 n+1\right)
$$

with bounded coefficients, i.e.

$$
\sigma_{i} \in L^{\infty}\left(R^{2 n+1}\right) \quad(i=1, \ldots, 2 n+1)
$$

such that, for some $1 \leq h \leq n$ and every $k \geq 1$,

$$
\rho X_{h}\left(\sigma_{2 n+1}\right) \in L^{q}\left(A_{k}\right) \quad \text { and } \quad \rho X_{h+n}\left(\sigma_{2 n+1}\right) \in L^{q}\left(A_{k}\right)
$$

with

$$
\left\|\rho X_{h}\left(\sigma_{2 n+1}\right)\right\|_{L^{\bullet}\left(A_{k}\right)} \leq \Lambda\left|A_{k}\right|^{\frac{1}{q}} \quad \text { and } \quad\left\|\rho X_{h+n}\left(\sigma_{2 n+1}\right)\right\|_{L^{\natural}\left(A_{k}\right)} \leq \Lambda\left|A_{k}\right|^{\frac{1}{q}}
$$

(D) $g$ is a bounded measurable function such that

$$
\rho g \in L^{q}\left(A_{k}\right), \quad \rho X_{h} g \in L^{q}\left(A_{k}\right), \quad \rho X_{h+n} g \in L^{q}\left(A_{k}\right)
$$

with

$$
\begin{aligned}
\|\rho g\|_{L^{\natural}\left(A_{k}\right)} & \leq \Lambda\left|A_{k}\right|^{\frac{1}{q}} \\
\left\|\rho X_{h} g\right\|_{L^{\bullet}\left(A_{k}\right)} & \leq \Lambda\left|A_{k}\right|^{\frac{1}{q}} \\
\left\|\rho X_{h+n} g\right\|_{L^{\natural}\left(A_{k}\right)} & \leq \Lambda\left|A_{k}\right|^{\frac{1}{q}} .
\end{aligned}
$$




\section{The Heisenberg group}

In this Section we recall the main properties of the Heisenberg group that we will use later. For more details see [17].

The space $\mathbb{R}^{2 n+1}$, whose elements we denote by $x=\left(x_{1}, \ldots, x_{n}, y_{1}, \ldots, y_{n}, t\right)$, equipped with the multiplication law

$$
x \cdot x^{\prime}=\left(x_{1}+x_{1}^{\prime}, \ldots, x_{n}+x_{n}^{\prime}, y_{1}+y_{1}^{\prime}, \ldots, y_{n}+y_{n}^{\prime}, t+t^{\prime}+\sum_{i=1}^{n}\left(x_{i}^{\prime} y_{i}-x_{i} y_{i}^{\prime}\right)\right)
$$

is a group whose identity is the origin and where the inverse is given by

$$
x^{-1}=\left(-x_{1}, \ldots,-x_{n},-y_{1}, \ldots,-y_{n},-t\right) \text {. }
$$

The space $\mathbb{R}^{2 n+1}$ with the structure (2.1) is the Heisenberg group, denoted by $H^{n}$. The non-isotropic dilations

$$
\delta \circ x=\left(\delta x_{1}, \delta x_{n}, \delta y_{1}, \delta y_{n}, \delta^{2} t\right) \quad\left(\delta \in \mathbb{R}, x \in H^{n}\right)
$$

are automorphisms of $H^{n}$. The non-negative function

$$
\rho(x)=\left(\sum_{i=1}^{n}\left(x_{i}^{2}+y_{i}^{2}\right)^{2}+t^{2}\right)^{\frac{1}{4}}
$$

is a norm for the Heisenberg group, in particular it is homogeneous of degree 1 with respect to the dilations $(2.2)$, i.e.

$$
\rho(\delta \circ x)=|\delta| \rho(x)
$$

for every $x \in H^{n}$ and $\rho \in \mathbb{R}$. Moreover, there exist positive constants $c_{1}$ and $c_{2}$ such that

$$
c_{1}|x|<\rho(x)<c_{2}|x|^{\frac{1}{2}}
$$

for every $x \in H^{n}$, where $|x|$ denotes the Euclidean norm in $\mathbb{R}^{2 n+1}$.

By (2.4) and (2.5) it follows that the function $d$ defined by

$$
d\left(x, x^{\prime}\right)=\rho\left(x^{\prime-1} \cdot x\right)
$$

is a distance in $H^{n}$, topologically equivalent to the Euclidean one and left invariant with respect to the law (2.1). By using the distance $d$ we define the intrinsic balls, spheres and neighborhoods of infinity

$$
\begin{aligned}
& B_{R}(x)=\left\{x^{\prime} \in H^{n}: d\left(x, x^{\prime}\right)<R\right\} \\
& S_{R}(x)=\left\{x^{\prime} \in H^{n}: d\left(x, x^{\prime}\right)=R\right\} \\
& D_{R}(x)=\mathbb{R}^{2 n+1} \backslash B_{R}(x) .
\end{aligned}
$$


In the following, for brevity, we will set

$$
B_{R}=B_{R}(0), \quad S_{R}=S_{R}(0), \quad D_{R}=D_{R}(0)
$$

The Lebesgue measure $d x=d x_{1} \cdots d x_{n} \cdot d y_{1} \cdots d y_{n} \cdot d t$ is invariant with respect to the translations (2.1), so that for any $x \in H^{n}$ and $R>0$ we have

$$
\left|B_{R}(x)\right|=\left|B_{R}\right| \text {. }
$$

Since the Jacobian of the dilations (2.2) is given by

$$
J_{\delta}=\delta^{2 n+2}
$$

from (2.9) and (2.10) it follows that for every $x \in H^{n}$ and $R>0$

$$
\left|B_{R}(x)\right|=R^{2 n+2}\left|B_{1}\right| \text {. }
$$

In our context it is convenient to look for a basis of vector fields invariant with respect to the translations (2.1). Such a basis is given by

$$
\begin{aligned}
X_{i} & = \begin{cases}\frac{\partial}{\partial x_{i}}+2 y_{i} \frac{\partial}{\partial t} & \text { for } i=1, \ldots, n \\
\frac{\partial}{\partial y_{i-n}}+2 x_{i-n} \frac{\partial}{\partial t} & \text { for } i=n+1, \ldots, 2 n\end{cases} \\
T & =\frac{\partial}{\partial t} .
\end{aligned}
$$

For the vector fields (2.12) we have the commutative law

$$
\left[X_{i}, X_{i+n}\right]=-4 T \quad \text { for every } i=1, \ldots, n
$$

while the other commutators vanish. We recall that a commutator of two vector fields $V_{1}$ and $V_{2}$ is the new vector field given by

$$
\left[V_{1}, V_{2}\right]=V_{1} V_{2}-V_{2} V_{1} \text {. }
$$

Therefore, $X_{1}, \ldots, X_{2 n}$ are a basis for the Lie algebra of the vector fields invariant with respect to (2.1). Moreover, they are homogeneous of degree 1 with respect to the dilations (2.2), whereas, by (2.13), $T$ is homogeneous of degree 2, i.e.

$$
X_{i}(u(\delta \circ x))=\delta\left(\left(X_{i} u\right)(\delta \circ x)\right) \quad(i=1, \ldots, 2 n)
$$

and

$$
T(u(\delta \circ x))=\delta^{2}((T u)(\delta \circ x))
$$

Given an open set $\Omega \subseteq \mathbb{R}^{2 n+1}$, we denote by $S^{2}(\Omega)$ the Sobolev-type space of the functions $u \in L^{2}(\Omega)$, such that the distribution derivatives $X_{i} u \in L^{2}(\Omega)$ for $i=$ $1, \ldots, 2 n$. The norm in $S^{2}(\Omega)$ is given by

$$
\left\||u \||^{2}=\int_{\Omega}\left(|u|^{2}+\sum_{i=1}^{2 n}\left|X_{i} u\right|^{2}\right) d x .\right.
$$

The closure of $C_{0}^{\infty}(\Omega)$ in the above norm is denoted by $\stackrel{\circ}{ }^{2}(\Omega)$. By. $S_{\text {loc }}^{2}(\Omega)$ we mean the set of functions $u \in S^{2}\left(\Omega^{\prime}\right)$ for every $\Omega^{\prime} \subset \subset \Omega$.

We recall the following properties of the above Sobolev spaces. 

have

Poincaré Inequality (see [11]). For every $B_{R}(x) \subset \mathbb{R}^{2 n+1}$ and $u \in \dot{S}^{2}\left(B_{R}(x)\right.$ ) we

$$
\int_{B_{R}(x)}|u|^{2} d x \leq c R^{2} \int_{B_{R}(x)} \sum_{j=1}^{2 n}\left|X_{j} u\right|^{2} d x .
$$

Sobolev Inequality (see [12]). There exists $S>0$ such that for every $u \in$ $S^{2}\left(\mathbb{R}^{2 n+1}\right)$ we have

$$
\left(\int_{\mathbb{R}^{2 n+1}}|u|^{2^{*}} d x\right)^{\frac{2}{2^{*}}} \leq S \int_{\mathbb{R}^{2 n+1}} \sum_{j=1}^{2 n}\left|X_{j} u\right|^{2} d x
$$

where $2^{*}=\frac{2 n+2}{n}$.

Compact Embedding (see [6]). For every bounded domain $\Omega$ the Sobolev space $\stackrel{\circ}{S}^{2}(\Omega)$ is compactly embedded into $L^{p}(\Omega)$, for every $p<2^{*}$.

\section{Dirichlet problems and maximum principles}

Let $\Omega$ be an open subset of $\mathbb{R}^{2 n+1}$. We consider the differential operator

$$
L u=-\sum_{i, j=1}^{2 n} X_{j}^{*}\left(a_{i j}(x) X_{i} u+d_{j}(x) u\right)+\sum_{i=1}^{2 n} b_{i}(x) X_{i} u+c(x) u
$$

whose associated bilinear form is given by

$$
a(u, v)=\int_{\Omega}\left(\sum_{i, j}\left(a_{i j} X_{i} u X_{j} v+b_{i}\left(X_{i} u\right) v+d_{j} u X_{j} v\right)+c u v\right) d x
$$

for $u \in S_{\text {loc }}^{2}(\Omega)$ and $v \in \stackrel{\circ}{S}^{2}(\Omega)$.

Let $f_{j} \in L^{2}(\Omega)(j=1, \ldots, 2 n)$ and $f \in L^{2^{*^{\prime}}}(\Omega)$.

Definition 3.1. We say that the function $u \in S_{\text {loc }}^{2}(\Omega)$ is a local solution of the equation

$$
L u=-\sum_{j=1}^{2 n} X_{j}^{*} f_{j}+f
$$

in $\Omega$, if for every $\varphi \in \stackrel{\circ}{S}^{2}(\Omega)$ we have

$$
a(u, \varphi)=\int_{\Omega}\left(\sum_{j} f_{j} X_{j} \varphi+f \varphi\right) d x .
$$

When $u \in S^{2}(\Omega)$ or $u \in \stackrel{\circ}{S}^{2}(\Omega)$ ), we say that $u$ is a solution or a solution vanishing on the boundary, respectively, of the equation (3.3). For brevity, when $f_{j} \equiv f \equiv 0$ ( $i=$ $1, \ldots, 2 n$ ), we say that $u$ is a local solution or a solution, respectively, of (the operator) $L$.

We start recalling some results concerning the operator $L$ when $\Omega$ is a bounded domain in $\mathbb{R}^{2 n+1}$. In the following we will assume that $L$ satisfies condition (A) on $\Omega$, $b_{i} \in L^{q}(\Omega)$ and $d_{i} \in L^{q}(\Omega) \quad(i=1, \ldots, 2 n), c \in L^{\frac{q}{2}}(\Omega)$ with $q>2 n+2$. 
Proposition 3.2. Let $T$ be a linear bounded operator on $\dot{S}^{2}(\Omega), \Omega \subset \mathbb{R}$ and $\sigma_{0} \in \mathbb{R}$. Then, whenever $\sigma_{0}$ is sufficiently large or $\operatorname{diam} \Omega$ is sufficiently small, the equation

$$
L u+\sigma_{0} u=T
$$

admits exactly one solution $u_{\sigma_{0}, T} \in \stackrel{\circ}{S}^{2}(\Omega)$. In particular this holds for the equation

$$
L u+\sigma_{0} u=-\sum_{j=1}^{2 n} X_{j}^{*} f_{j}+f
$$

where $f_{j} \in L^{2}(\Omega) \quad(j=1, \ldots, 2 n)$ and $f \in L^{2^{*^{\prime}}}(\Omega)$. Furthermore, the map $L_{\sigma_{0}}^{-1}: T \mapsto$ $u_{\sigma_{0}, T}$ is continuous.

Proof. See [16]

Proposition 3.3 (Caccioppoli Inequality). Let $\Omega^{\prime} \subset \subset \Omega$. Then there exists a positive constant $k$ such that, for every local solution or non-negative subsolution $u$ of $L$ in $\Omega$, we have

$$
\sum_{j=1}^{2 n}\left\|X_{j} u\right\|_{L^{2}\left(\Omega^{\prime}\right)} \leq k\|u\|_{L^{2}(\Omega)} .
$$

Proof. See [16: Lemma 5.2] or [13: Lemma 4.4]

Proposition 3.4 (Local Harnack Inequality). For any compact $K \subset \Omega$ there exists a positive constant $C=C\left(M, \mu, K, \Omega,\left\|b_{i}\right\|_{L^{q}},\left\|d_{i}\right\|_{L^{q}},\|c\|_{L^{2}}\right)$ such that, for any positive local solution $u$ of $L$ in $\Omega$,

$$
C u(x)<u(y)
$$

holds for every $x, y \in K$.

Proof. For every $B_{R}(x) \subset \Omega$ with $R$ sufficiently small, we can prove the following:

Step 1: For every $p>0$ there exists a positive constant $c_{p}$ such that

$$
\sup _{B_{R}(x)} u \leq c_{p}\left(f_{B_{2 R}(x)}|u|^{p} d x\right)^{\frac{1}{p}}
$$

(see [13: Theorem 4.2]).

Step 2: From Step 1 it follows that for every $q<0$ there exists a positive constant $c_{q}$ such that

$$
\inf _{B_{R}(x)} u \geq c_{q}\left(f_{B_{2 R}(x)}|u|^{q} d x\right)^{\frac{1}{q}}
$$

(see [13: Proposition 4.8]). 
Step 3: There exist $\delta \in(0,1)$ and $A \geq 1$ such that

$$
f_{B_{2 R}(x)}|u|^{\delta} d x \cdot f_{B_{2 R}(x)}|u|^{-\delta} d x \leq A
$$

(see [13: Proposition 5.2]).

The proof follows from Steps 1-3 and from compactness arguments

Remark 3.5. We can prove that Step 1 still holds for non-negative solutions and positive subsolutions and Step 3 for positive supersolutions (see [16: Section 8]).

Proposition 3.6 (Hölder continuity). For any compact $K \subset \Omega$ there exist positive constants $\delta, c, c^{\prime}$ and $0<\lambda<1$ such that, for any local solution $u$ of $L$ in $\Omega$,

$$
|u(x)-u(y)| \leq c^{\prime}\|u\|_{L^{2}} d(x, y)^{\lambda} \leq c\|u\|_{L^{2}}|x-y|^{\frac{\lambda}{2}}
$$

holds for every $x, y \in K$ with $d(x, y)<\delta$.

Proof. See [13: Theorem 5.6]

Remark 3.7. The previous results still hold under the weaker assumption $b_{i} \epsilon$ $L^{2 n+2}(\Omega) \quad(i=1, \ldots, 2 n)$, but in this case the constants involved in the inequalities depend on $b_{i}$ and not only on $\left\|b_{i}\right\|_{L^{a}}$. In the next section, in order to prove a uniform local Harnack inequality, we need the constant $C$ of inequality (3.8) to depend only on $\left\|b_{i}\right\|_{L^{q}}$, therefore we assume $b_{i} \in L^{q}$ from now on.

Following [10: Theorem 8.3], we will prove the existence for the Dirichlet problem via the Maximum Principle. In order to formulate it we need the following notion of supremum on the boundary for a function $u \in S_{\text {loc }}^{2}(\Omega)$ :

$$
\sup _{\partial \Omega} u=\inf \{k \in \mathbb{R}: u \leq k\}
$$

$\mathrm{x}$ where by $u \leq k$ on $\partial \Omega$ and $u \geq k$ on $\partial \Omega$ we mean $(u-k)^{+} \in \dot{S}^{2}(\Omega)$ and $(u-k)^{-} \epsilon$ $\dot{S}^{2}(\Omega)$, respectively. If $u \leq k$ and $u \geq k$ on $\partial \Omega$, we say that $u=k$ on $\partial \Omega$.

Weak Maximum Principle. Let $u$ be a local solution of $L$ in a bounded domain $\Omega$. Then

$$
\sup _{\Omega} u \leq \max \left(0, \sup _{\partial \Omega} u\right) \quad \text { and } \quad \min \left(0, \inf _{\partial \Omega} u\right) \leq \inf _{\Omega} u
$$

where $\inf _{\partial \Omega} u=-\sup _{\partial \Omega}(-u)$.

In the following we will need also the

Strong Maximum Principle. Let $u$ be a non-negative local solution of $L$ on a domain $\Omega$. Then either $u \equiv 0$ or $u>0$ on $\Omega$.

From now on we will assume that the operator $L$ satisfies the Weak Maximum Principle. 
Proposition 3.8. Let $u \in \dot{S}^{2}(\Omega)$ be a solution of L. Then $u \equiv 0$.

From Proposition 3.8 and the Fredholm alternative there follows an existence and uniqueness theorem for the Dirichlet problem in bounded domains, analogous to [10: Theorem 8.3]. We give here the simplified version that we will use later.

Proposition 3.9 (Existence Theorem). For every constant $k$ there exists exactly one solution $u \in S^{2}(\Omega)$ of

$$
\left.\begin{array}{rll}
L u=0 & \text { on } & \Omega \\
u=k & \text { on } & \partial \Omega .
\end{array}\right\}
$$

Proof. The problem (3.10) is equivalent to the problem

$$
L w=k\left(\sum_{j=1}^{2 n} X_{j}^{*} d_{j}-c\right) \quad\left(w \in \stackrel{\circ}{S}^{2}(\Omega)\right)
$$

where $w=u-k$. Let $\sigma_{0}$ be sufficiently large so that the equation

$$
L v+\sigma_{0} v=k\left(\sum_{j=1}^{2 n} X_{j}^{*} d_{j}-c\right)
$$

admits exactly one solution $v \in \dot{S}^{2}(\Omega)$. Then the equation (3.11) is equivalent to the equations

$$
\begin{aligned}
& \left(L w+\sigma_{0} w\right)-\sigma_{0} w=k\left(\sum_{j=1}^{2 n} X_{j}^{*} d_{j}-c\right) \\
& w-\sigma_{0} L_{\sigma_{0}}^{-1} I w=k L_{\sigma_{0}}^{-1}\left(\sum_{j=1}^{2 n} X_{j}^{*} d_{j}-c\right)
\end{aligned}
$$

where $I$ is the compact embedding of $\dot{S}^{2}(\Omega)$ into $L^{2}(\Omega)$ and $L^{-1} \sigma_{0}$ is the map defined in Proposition 3.2. By the Compact Embedding Property of Sobolev spaces and Proposition $3.2, \sigma_{0} L_{\sigma_{0}}^{-1} I$ is a compact operator from $\dot{S}^{2}(\Omega)$ into itself so that, by Proposition 3.8 and the Fredholm alternative, there exists exactly one solution of (3.14) and so of (3.10)

Theorem 3.10. Let $L$ satisfy assumption (A) in $\mathbb{R}^{2 n+1}, b_{i} \in L_{\text {loc }}^{q}\left(\mathbb{R}^{2 n+1}\right)$ and $d_{i} \in L_{\text {loc }}^{q}\left(\mathbb{R}^{2 n+1}\right) \quad(i=1, \ldots, 2 n), c \in L_{\text {loc }}^{\frac{8}{2}}\left(\mathbb{R}^{2 n+1}\right)$ with $q>2 n+2$. Let us assume that $L$ satisfies also the Strong Maximum Principle. Then there exists at least a positive local solution $u \in S_{\text {loc }}^{2}\left(\mathbb{R}^{2 n+1}\right)$ of $L$.

Proof. Let $R_{k} \nearrow+\infty$. By Proposition (3.9) there exists a sequence $\left\{v_{k}\right\}$ of positive solutions of the problems

$$
\left.\begin{array}{rll}
L v_{k}=0 & \text { on } & B_{R_{k}} \\
v_{k}=1 & \text { on } & \partial B_{R_{k}}
\end{array}\right\}
$$


Let us consider the sequence of solutions $u_{k}=\frac{v_{k}}{v_{k}(0)}$. Fixed $N \geq 1$, the sequence $\left\{u_{k}\right\}$ converges in $\bar{B}_{R_{N}}$ to a continuous positive function $u \in S^{2}\left(\bar{B}_{R_{N}}\right)$. Actually, by the local Harnack inequality, there exists $c_{N}>0$ such that for every $k>N$

$$
c_{N} u_{k}(x) \leq u_{k}(0)=1
$$

Hence

$$
\left\|u_{k}\right\|_{L^{2}\left(\bar{B}_{R_{N}}\right)} \leq\left\|u_{k}\right\|_{L^{\infty}\left(\bar{B}_{R_{N}}\right)}\left|B_{R_{N}}\right|^{\frac{1}{2}} \leq \frac{\left|B_{R_{N}}\right|^{\frac{1}{2}}}{c_{N}}
$$

By (3.16) and (3.9) the sequence $\left\{u_{k}\right\}$ is bounded in $C\left(\bar{B}_{R_{N}}\right)$ and uniformly equicontinuous. So by Ascoli's Theorem it converges (up to a subsequence) to a continuous function $u$, that is a positive local solution of $L$ in $\mathbb{R}^{2 n+2}$. Actually, by the Caccioppoli inequality and (3.16), the sequence $\left\{\left\|X_{i} u_{k}\right\|_{L^{2}\left(B_{R_{N}}\right)}\right\}$ is bounded, so $\left\{u_{k}\right\}$ converges in every $\bar{B}_{R_{N}}$ to $u$ also in the norm (2.17)

We give now two sufficient conditions for the Maximum Principles to hold.

Proposition 3.11. Let $-\sum_{i, j=1}^{2 n} X_{j}^{*} d_{j}+c$ be positive in the sense of distibutions on $\Omega$, i.e.

$$
\int_{\Omega}\left(\sum_{j} d_{j} X_{j} \varphi+c \varphi\right) d x \geq 0 \quad \text { for every } 0 \leq \varphi \in \dot{S}^{2}(\Omega)
$$

\section{Then the Weak Maximum Principle holds.}

Proof. For sake of completeness we give the proof, that is analogous to that of [10: Theorem 8.1]. Let $u$ be a local solution of $L$. Let us suppose that there exist constants $k$ such that

$$
\max \left(0, \sup _{\partial \Omega} u\right)<k<\sup _{\Omega} u
$$

Set $v_{k}=(u-k)^{+}$. Since $v_{k} \in \dot{S}^{2}(\Omega)$, we have

$$
\begin{aligned}
0 & =a\left(u, v_{k}\right) \\
& =\int_{\Omega}\left(\sum_{j}\left(a_{i, j} X_{i} u X_{j} v_{k}+\left(b_{i}-d_{i}\right)\left(X_{i} u\right) v_{k}+d_{i} X_{i}\left(u v_{k}\right)\right)+c u v_{k}\right) d x .
\end{aligned}
$$

From (3.17), (3.19) and $u v_{k} \geq 0$ it follows

$$
\int_{\Omega} \sum_{i, j} a_{i j} X_{i} u X_{j} v_{k} d x \leq \int_{\Omega} \sum_{i}\left(d_{i}-b_{i}\right)\left(X_{i} u\right) v_{k} d x
$$


Hence, from the assumptions on the coefficients of $L$ and from the Sobolev inequality, it follows

$$
\begin{aligned}
\mu \sum_{j}\left\|X_{j} v_{k}\right\|_{L^{2}}^{2} & \leq \int_{\Omega} \sum_{i, j} a_{i j} X_{i} v_{k} X_{j} v_{k} d x \\
& =\int_{\Omega} \sum_{i, j} a_{i j} X_{i} u X_{j} v_{k} d x \\
& \leq \int_{\Omega} \sum_{i}\left(d_{i}-b_{1}\right)\left(X_{i} u\right) v_{k} d x \\
& =\int_{\Omega} \sum_{i}\left(d_{i}-b_{i}\right)\left(X_{i} v_{k}\right) v_{k} d x \\
& \leq K \sum_{i}\left|\operatorname{supp} X_{i} v_{k}\right|^{\frac{q-2 n-2}{(2 n+2)}} \sum_{i}\left\|b_{i}-d_{i}\right\|_{L^{q}} \sum_{i}\left\|X_{i} v_{k}\right\|_{L^{2}}^{2} .
\end{aligned}
$$

Therefore for every $k$ that satisfies (3.18) we have

$$
\left|\operatorname{supp} X_{i} v_{k}\right| \geq \mu^{\frac{q(2 n+2)}{q-2 n-2}}\left(\sum_{i}\left\|b_{i}-d_{i}\right\|_{L^{q}}\right)^{\frac{q(2 n+2)}{q-2 n-2}}
$$

So $u$ attains its supremum on a set of positive measure where $X_{i} u \neq 0$. This contradicts the existence of $k$ satisfying (3.18)

Proposition 3.12. Let $L$ satisfy assumption (3.17). Then the Strong Maximum Principle holds.

Proof. Let $u$ be a non-negative local solution of $L$. By assumption (3.17), $(u+\varepsilon)$ is a positive local supersolution of $L$ and $(u+\varepsilon)^{q} \quad(q<0)$ a positive local subsolution of $\widetilde{L}$ where

$$
\widetilde{L}=-\sum_{i, j=1}^{2 n} X_{j}^{*}\left(a_{i j}(x) X_{i} u+q d_{j}(x) u\right)+\sum_{i=1}^{2 n}\left(b_{i}(x)+(q-1) d_{i}(x)\right) X_{i} u+q c(x) u
$$

so that Steps 2 and 3 of Proposition 3.4 still hold for $(u+\varepsilon)$. By the monotone convergence theorem they also hold for $u$. Therefore, by inequality (3.8) applied to $u$, it follows that either $u \equiv 0$ or $u>0$

Proposition 3.13. Let there exists a positive local solution $w$ of $L$ in $\Omega$, positive on $\partial \Omega$ and such that $X_{i} w \in L^{q}(\Omega)$ with $q>2 n+2$, for every $i=1, \ldots, 2 n$. Then $L$ satisfies the Weak and Strong Maximum Principles.

Proof. Let $u$ be a local solution of $L$ non-negative on $\partial \Omega$. Set $v=\frac{u}{w}$. For every $\varphi \in \stackrel{\circ}{S}^{2}(\Omega)$ we have

$$
0=a(u, \varphi)=a(v w, \varphi)=a(w, v \varphi)+\tilde{a}(v, \varphi)=\tilde{a}(v, \varphi)
$$


where

$$
\tilde{a}(v, \varphi)=\int_{\Omega}\left(\sum_{i, j} w a_{i j} X_{i} v X_{j} \varphi+\left(\sum_{i}\left(w b_{i}-w d_{i}\right)-\sum_{i, j} a_{i j} X_{j} w\right)\left(X_{i} v\right) \varphi\right) d x
$$

By the assumptions on $w$, the coefficients of $\tilde{\boldsymbol{a}}$ satisfy the assumptions of Proposition 3.11. Therefore from $v \geq 0$ on $\partial \Omega$ it follows that $v$, and so $u$, are non-negative on $\Omega$ and also that they are either positive or identically equal to zero

For a thorough discussion of the Maximum Principle see also [4] and the included references.

\section{Liouville Theorems}

In this section we prove Liouville's theorems assuming $L$ to be a Fuchsian-type operator.

Definition 4.1. We say that the operator defined in (1.2) is a Fuchsian operator in the weak sense, if there exist $0<a<1<b$ and $R_{k} \nearrow+\infty$ such that $L$ satisfies assumptions (A) and (B).

From now on we will assume that $L$ is a Fuchsian operator in the weak sense.

Proposition 4.2 (Uniform local Harnack inequality). There exists a positive constant $C$ depending on $\mu, M, \Lambda, a, b, a^{\prime}$ and $b^{\prime}$ such that, for every $k \geq 1$ and every positive solution $u$ of $L$ in $A_{k}$, we have

$$
C u(x)<u(y) \quad \text { for all } x, y \in A_{k}^{\prime}
$$

where $A_{k}^{\prime}$ is the annulus

$$
A_{k}^{\prime}=\left\{x \in \mathbb{R}^{2 n+1}: a^{\prime} R_{k}<\rho(x)<b^{\prime} R_{k}\right\}
$$

and $a<a^{\prime}<1<b^{\prime}<b$.

Proof. Let $u$ be a positive solution of $L$ in $A_{k}$. Let us define in the annulus

$$
A=\left\{\xi \in \mathbb{R}^{2 n+1}: a<\rho(\xi)<b\right\}
$$

the function

$$
w(\xi)=w_{k}(\xi)=u\left(R_{k} \circ \xi\right)
$$

By the homogeneity of $X_{j}$ with respect to the dilations (2.2) and by (2.10), we have for 
every $\varphi \in \stackrel{\circ}{S}^{2}\left(A_{k}\right)$

$$
\begin{aligned}
0= & \int_{A_{k}}\left(\sum _ { i , j } \left(a_{i j}(x) X_{i} u(x) X_{j} \varphi(x)+b_{i}(x)\left(X_{i} u(x)\right) \varphi(x)\right.\right. \\
& \left.\left.+d_{j}(x) u(x) X_{j} \varphi(x)\right)+c(x) u(x) \varphi(x)\right) d x \\
= & \int_{A}\left(\sum _ { i , j } \left(\frac{1}{R_{k}^{2}} a_{i j}\left(R_{k} \circ \xi\right) X_{i} w(\xi) X_{j} \psi(\xi)\right.\right. \\
& \left.+\frac{1}{R_{k}} b_{i}\left(R_{k} \circ \xi\right)\left(X_{i} w(\xi)\right) \psi(\xi)+\frac{1}{R_{k}} d_{j}\left(R_{k} \circ \xi\right) w(\xi) X_{j} \psi(\xi)\right) \\
& \left.+c\left(R_{k} \circ \xi\right) w(\xi) \psi(\xi)\right) R_{k}^{2 n+2} d \xi \\
= & R_{k}^{2 n} \int_{A}\left(\sum_{i, j}\left(a_{i j}^{k} X_{i} w X_{j} \psi+b_{i}^{k}\left(X_{i} w\right) \psi+d_{j}^{k} w X_{j} \psi\right)+c^{k} w \psi\right) d \xi
\end{aligned}
$$

where $a_{i j}^{k}(\xi)=a_{i j}\left(R_{k} \circ \xi\right), b_{i}^{k}(\xi)=R_{k} b_{i}\left(R_{k} \circ \xi\right), d_{i}^{k}(\xi)=R_{k} d_{i}\left(R_{k} \circ \xi\right) \quad(i=1 \ldots, 2 n)$, $c^{k}(\xi)=R_{k}^{2} c\left(R_{k} \circ \xi\right)$ and $\psi(\xi)=\varphi\left(R_{k} \circ \xi\right)$. Since for every $\psi \in \dot{S}^{2}(A)$ we have $\psi(\xi)=\varphi\left(R_{k} \circ \xi\right)$ where $\varphi \in \dot{S}^{2}\left(A_{k}\right)$ is given by $\varphi(x)=\psi\left(\frac{1}{R_{k}} \circ x\right)$, from (4.3) it follows that $w$ is a positive solution of $L_{k}$ in $A$, where

$$
L_{k} w=-\sum_{i, j=1}^{2 n} X_{j}^{*}\left(a_{i j}^{k}(\xi) X_{i} w+d_{j}^{k}(\xi) w\right)+\sum_{i=1}^{2 n} b_{i}^{k}(\xi) X_{i} w+c^{k}(\xi) w .
$$

By the homogeneity of $\rho$ with respect to the dilations (2.2), by (2.10), (1.5) and (2.11) it follows for every $i=1, \ldots, 2 n$ that

$$
\begin{aligned}
\int_{A}\left(b_{i}^{k}\right)^{q} d \xi & =\int_{A} R_{k}^{q} b_{i}\left(R_{k} \circ \xi\right)^{q} d \xi=\int_{A}\left(\frac{\rho\left(R_{k} \circ \xi\right)}{\rho(\xi)} \cdot b_{i}\left(R_{k} \circ \xi\right)\right)^{q} d \xi \\
& \leq \frac{1}{a^{q}} \int_{A}\left(\rho\left(R_{k} \circ \xi\right) b_{i}\left(R_{k} \circ \xi\right)\right)^{q} d \xi=\frac{1}{a^{q} R^{2 n+2}} \int_{A_{k}}\left(\rho(x) b_{i}(x)\right)^{q} d x \\
& \leq \frac{1}{a^{q}} \Lambda^{q} \frac{\left|A_{k}\right|}{R_{k}^{2 n+2}} \leq \frac{b^{2 n+2}-a^{2 n+2}}{a^{q}} \Lambda^{q}\left|B_{1}\right| .
\end{aligned}
$$

In the same way we can prove for every $k \geq 1$ and every $j=1 \ldots, 2 n$ that

$$
\left\|d_{j}^{k}\right\|_{L^{q}(A)}^{q} \leq \frac{b^{2 n+2}-a^{2 n+2}}{a^{q}} \Lambda^{q}\left|B_{1}\right|
$$

and

$$
\left\|c^{k}\right\|_{L^{\frac{q}{2}(A)}}^{\frac{2}{2}} \leq \frac{b^{2 n+2}-a^{2 n+2}}{a^{q}} \Lambda^{\frac{q}{2}}\left|B_{1}\right| .
$$

Let $x, y \in A_{k}^{\prime}$. Then $\xi=\frac{1}{R_{k}} \circ x$ and $\eta=\frac{1}{R_{k}} \circ y$ belong to $A^{\prime}$. By the local Harnack inequality (3.8) there exists $C$, depending only on $a, b, a^{\prime}, b^{\prime}$ and on $\mu, M, \Lambda$, but not on $k$, such that

$$
C w_{k}(\xi)<w_{k}(\eta)
$$

By the definition of $w$, from (4.8) it follows (4.1) 
Corollary 4.3. Let $u$ and $v$ be two positive local solutions of $L$ in $A_{k}$. Then we have

$$
C^{2} \frac{u(x)}{v(x)}<\frac{u(y)}{v(y)} \quad \text { for all } x, y \in A_{k}^{\prime}
$$

where $C$ is the same constant appearing in the inequality (4.1).

Proof. From $C u(x)<u(y)$ and $C v(y)<v(x)$ it follows (4.9)

From now on we will assume that $L$ satisfies the Weak and Strong Maximum Principles.

Proposition 4.4. Let $u$ and $v$ be two positive local solutions of $L$ in $D_{R}$ for some $R>0$. Then there exists

$$
\lim _{\rho(x) \rightarrow+\infty} \frac{u(x)}{v(x)}
$$

Proof. Let $a_{k}=\min _{S_{R_{k}}} \frac{u(x)}{v(x)}$ and $b_{k}=\max _{S_{R_{k}}} \frac{u(x)}{v(x)}$. Set $\alpha_{k}=\min \left(a_{k-1}, a_{k+1}\right)$ and $\beta_{k}=\max \left(b_{k-1}, b_{k+1}\right)$. The functions $u-\alpha_{k} v$ and $\beta_{k} v-u$ are solutions of $L$ in

$$
G_{k}=\left\{x \in \mathbb{R}^{2 n+1}: R_{k-1}<\rho(x)<R_{k+1}\right\},
$$

non-negative on $\partial G_{k}$. Hence by the Maximum Principles they are positive in $G_{k}$ or they are identically equal to zero. Therefore either $u(x)=\alpha_{k} v(x)$ on $S_{R_{k}}$ for some $R_{k}$ and so on $D_{R}$, or the sequence $\left\{a_{k}\right\}$ is definitively strictly monotone. Actually, if $a_{k} \leq a_{k-1}$, by the Strong Maximum Principle saying that $a_{k}>\alpha_{k}$, it follows $a_{k+1}<a_{k}$. In the same way we can prove that either $b_{k}$ is constant or it is definitively monotone.

Let $a=\lim _{k \rightarrow+\infty} a_{k}$ and $b=\lim _{k \rightarrow+\infty} b_{k}$. Since $a_{k} \leq b_{k}$ if $a=+\infty$ we are done. If $a<+\infty$, by the Harnack inequality it still follows that $a=b$. Actually, let $x_{k}, y_{k} \in S_{R_{k}}$ be such that $\frac{u\left(x_{k}\right)}{v\left(x_{k}\right)}=b_{k}-\varepsilon_{k}$ and $\frac{u\left(y_{k}\right)}{v\left(y_{k}\right)}=a_{k}+\eta_{k}$ with $\varepsilon_{k} \searrow 0$ and $\eta_{k} \searrow 0$. Then by inequality (4.9) applied to the positive solutions $u-\alpha_{k} v$ and $v$ it follows

$$
C^{2}\left(b_{k}-\varepsilon_{k}-\alpha_{k}\right) \leq\left(a_{k}+\eta_{k}-\alpha_{k}\right)
$$

By passing to the limit we have $0 \leq C^{2}(b-a) \leq 0$. Since $\alpha_{k} \leq \frac{u(x)}{v(x)} \leq \beta_{k}$ holds for every $x \in G_{k}$, the existence of the limit (4.10) follows from $a=b$

As a by-product of this limit theorem we obtain a Liouville theorem for the operator $L$.

Theorem 4.5 (Liouville Theorem). There exists a unique, up to a constant, positive local solution of $L$ in $\mathbb{R}^{2 n+1}$.

Proof. The existence of at least one local solution is given by Theorem 3.10 . To prove the uniqueness it is sufficient to observe that if the solutions $u$ and $v$ in Proposition 4.4 are defined in $\mathbb{R}^{2 n+1}$, then by the Weak Maximum Principle the sequences $\left\{a_{k}\right\}$ and $\left\{b_{k}\right\}$ must be non-increasing and non-decreasing, respectively. This, toghether with $a=b$, yields $u(x) \equiv a v(x)$ on $\mathbb{R}^{2 n+1}$ 
From now on we will assume that $L$ is a Fuchsian operator, i.e. that it is a Fuchsian operator in the weak sense and there exists $R>0$ such that

$$
D_{R} \subset \cup A_{k}^{\prime} \text {. }
$$

Lemma 4.6. Let $u$ be a positive local solution of $L$ in $\mathbb{R}^{2 n+1}$. Then inf $u>0$.

Proof. Let us assume that $\inf _{\mathbb{R}^{2 n+1}} u=0$. Then, by the Strong Maximum Principle, a minimizing sequence $\left\{x_{i}\right\}$ must diverge, i.e. $\rho\left(x_{i}\right) \rightarrow+\infty$. Let $R_{i}=\rho\left(x_{i}\right)$, $a_{i}=\min _{S_{R_{i}}} u$ and $b_{i}=\max _{S_{R_{i}}} u$. By (4.11), for every $i$ there exists $k_{i}$ such that $S_{R_{i}} \subset A_{k_{i}}^{\prime}$. Since $\left\{a_{i}\right\}$ converges to zero, by the uniform Harnack inequality $\left\{b_{i}\right\}$ also converges to zero. Therefore, by the Weak Maximum Principle, for every $k_{i}$ and every $\varepsilon>0$ we have $\sup _{B_{R_{k_{i}}}} u<\varepsilon$, i.e. $u \equiv 0$ on $\mathbb{R}^{2 n+1}$

Theorem 4.7 (Liouville Property). Let $v$ be a local solution bounded below of $L$ in $\mathbb{R}^{2 n+1}$. Then either $v \equiv 0$ or $v$ is of constant sign. The same result holds if $v$ is bounded above.

Proof. Let $v>k$. If $k \geq 0$, we are done. If $k<0$, by Theorem 3.10 and Lemma 4.6, there exists a positive local solution $u>-k$ in $\mathbb{R}^{2 n+1}$. Then $v+u$ is a positive local solution, too. Therefore, by the Liouville Theorem, there exists $\lambda>0$ such that $v=(\lambda-1) u$. The second part of the proposition follows by observing that if $v<k$, then $-v$ is of constant sign

Remark 4.8. All the previous results still hold when we consider the operator

$$
\widetilde{L} u=L u+g(x) X_{0} u
$$

where $L, X_{0}$ and $g$ satisfy assumptions (A), (B), (C) and (D) of Section 1. Actually, by (2.13),

$$
X_{0}=\sum_{i=1}^{2 n} \sigma_{i} X_{i}-\frac{1}{4} \sigma_{2 n+1}\left[X_{h}, X_{h+1}\right]
$$

so that

$$
L u=-\sum_{i, j=1}^{2 n} X_{j}^{*}\left(\tilde{a}_{i j}(x) X_{i} u+d_{j}(x) u\right)+\sum_{i=1}^{2 n} \widetilde{b}_{i}(x) X_{i} u+c(x) u
$$

were $\tilde{a}_{i j}=a_{i j}$, when $(i, j) \neq(h, h+n),(h+n, h)$ while

$$
\tilde{a}_{h, h+n}=a_{h, h+n}-\frac{1}{4} g \sigma_{2 n+1} \quad \text { and } \quad \tilde{a}_{h+n, h}=a_{h+n, h}+\frac{1}{4} g \sigma_{2 n+1}
$$

and $\tilde{b}_{i}=b_{i}+g \sigma_{i}$, when $i \neq h, h+n$ while

$$
\tilde{b}_{h}=b_{h}+g \sigma_{h}-\frac{1}{4} g X_{h+n}\left(\sigma_{2 n+1}\right)-\frac{1}{4} \sigma_{2 n+1} X_{h+n} g
$$

and

$$
\tilde{b}_{h+n}=b_{h+n}+g \sigma_{h+n}+\frac{1}{4} g X_{h}\left(\sigma_{2 n+1}\right)+\frac{1}{4} \sigma_{2 n+1} X_{n} g .
$$

Acknowledgement. The authors would like to thank Prof. Umberto Mosco for suggesting the problem and for the several discussions during the preparation of this paper. 


\section{References}

[1] Berestycki, H. and L. Nirenberg: Asymptotic behaviour via the Harnack inequality. In: Nonlinear Analysis. A tribute in honour of G. Prodi (eds.: A. Marino and A. Ambrosetti). Pisa: Scuola Normale Superiore 1991, pp. 135 - 144.

[2] Birindelli, I., Capuzzo-Dolcetta, I. and A. Cutri: Liouville theorems for semilinear equations on the Heisenberg group. Ann. Inst. H. Poincaré - Analyse non linéaire (to appear).

[3] Biroli, M. and U. Mosco: A Saint Venant principle for Dirichlet forms on discontinuous media. Ann. Mat. Pura Appl. 168 (1995), 125 - 181.

[4] Chicco, M.: Generalizzazioni del principio di massimo e maggiorazione delle soluzioni per operatori ellittici di tipo variazionale. Boll. Unione Matematica Italiana 18-A (1981), 449 $-456$.

[5] De Cicco, D. and M. A. Vivaldi: Harnack inequalities for Fuchsian type weighted elliptic equation. Comm. Part. Diff. Equ. (to appear).

[6] Folland, G. B.: A fundamental solution for a subelliptic operator. Bull. Amer. Math. Soc. 79 (1973), 373 - 376.

(7) Garofalo, N. and E. Lanconelli: Existence and non existence results for semilnear equation on the Heisenberg group. Indiana Univ. Math. J. 41 (1992), 71 - 97.

[8] Gaveau, B.: Principe de moindre action, propagation de la chaleur et estimées souselliptiques sur certain groups nilpotents. Acta Math. 139 (1977), 95 - 153.

[9] Gilbarg, D. and J. Serrin: On isolated singularities of solutions of second order elliptic differential equations. J. Analyse Math. 4 (1956), $309-340$.

[10] Gilbarg, D. and N. S. Trudinger: Elliptic Partial Differential Equations of Second Order. Berlin: Springer-Verlag 1983.

[11] Jerison, D. s.: Poincaré inequality for vector fields satisfying Hörmander's condition. Duke Math. J. 53 (1986), $503-523$.

[12] Jerison, D. S. and J. M. Lee: Extremal for the Sobolev inequality on the Heisenberg group and CR Yamabe problem. J. Amer. Math. Soc. 1 (1988), 1- 13.

[13] Lancia, M. R. and M. V. Marchi: Harnack inequalities for nonsymmetric operator of Hörmander type with discontinuous coefficients. Adv. Math. Sci. Appl. (to appear).

[14] Landis, E. M. and V. S. Nadirashvili: Positive solutions of second order elliptic equations in unbounded domains. Math. USSR Sbornik 54 (1986), $129-134$.

[15] Pinchover, Y.: On positive Liouville's theorems and asymptotic behaviour of solutions of Fuchsian type elliptic operators. Ann. Inst. H. Poincaré. Analyse non linéaire 11 (1994), $313-341$.

[16] Stampacchia, G.: Les problèmes de Dirichlet pour les équations elliptiques du second ordre à coefficients discontinues. Ann. Inst. Fourier (Grenoble) 15 (1965), $189-258$.

[17] Stein, E. M.: Harmonic Analysis (Princeton Math. Series: Vol. 43). Princeton (N.J.): Univ. Press 1993. 